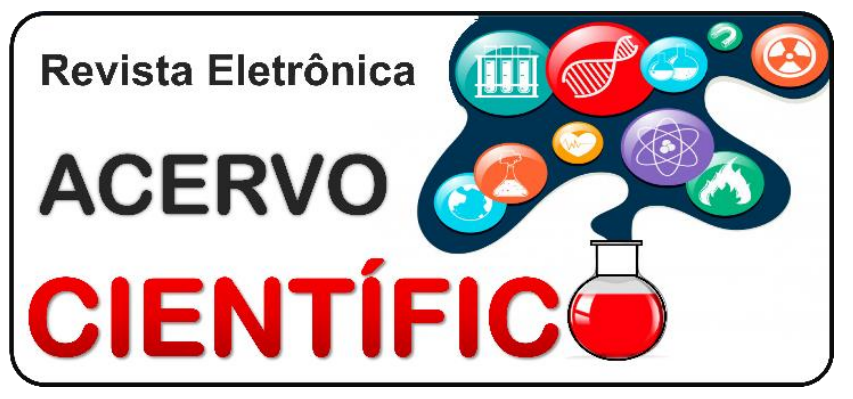

REVISÃO BIBLIOGRÁFICA

Recebido em: 11/2020

Aceito em: $12 / 2020$

Publicado em: 1/2021

\title{
Alternativas para substituição da valva aórtica em casos de estenose
}

\author{
Alternatives for substitution of aorthic valve in estenosis cases
}

\section{Alternativas de sustitución de válvula aórtica em casos de estenosis}

Hendel Lucas Pereira do Amaral ${ }^{1 *}$, Gabriel Porto Soares ${ }^{2}$, Camila Borges Ferreira ${ }^{3}$, Cecília Corrêa Fernandes $^{4}$, Dafne Dalledone Moura ${ }^{5}$, João Marcos Cavalcante Tavares ${ }^{6}$, Maria Karoline de Souza ${ }^{7}$, Maria Vitória do Nascimento Silva ${ }^{8}$, Nathália Santos Tigre $^{9}$, Priscila Pereira Albuquerque ${ }^{10}$.

\begin{abstract}
Resumo: Esse artigo buscou realizar uma revisão da literatura comparativa entre as técnicas de substituição da valva aórtica transcateter (TAVR) e de substituição da valva aórtica cirúrgica (SAVR), ambas utilizadas no tratamento da estenose aórtica (EA). Analisando as duas técnicas do ponto de vista do prognóstico e da morbimortalidade. A estenose aórtica consiste no estreitamento desta valva, é uma condição comum e decorrente de diversas etiologias, sendo que frequentemente evolui com hipertrofia do ventrículo esquerdo e diminuição da sua função. Atualmente, para o tratamento desta patologia, houve um aumento da utilização da TAVR, devido não somente a um avanço técnico-científico que possibilitou uma otimização da técnica, mas também em consequência do fato de apresentar menor incidência de algumas complicações. Apesar da SAVR ainda ser considerada o padrão ouro, a TAVR é menos invasiva e é mais indicada para pacientes com alto risco cirúrgico, além de apresentar eficácia e segurança semelhantes a SAVR e melhor prognóstico póscirurgia. Entretanto, é fundamental a análise de outras variáveis como risco, benefício e comorbidades do paciente antes de se optar pela melhor técnica de tratamento.
\end{abstract}

Palavras-chave: Estenose da valva aórtica, Substituição da valva aórtica transcateter, Substituição da valva aórtica cirúrgica.

\footnotetext{
Abstract: This article sought to conduct a comparative literature review between the transcatheter aortic valve replacement (TAVR) and surgical aortic valve replacement (SAVR) techniques, both used in the treatment of aortic stenosis $(\mathrm{AE})$, relating the two techniques from the point of view of prognosis, morbidity and mortality. Aortic stenosis consists of the narrowing of this valve, it is a common condition caused by several etiologies, and it often evolves with left ventricular hypertrophy and function decreased. Currently, for the treatment of this pathology, there has been an increase in the use of TAVR, due not only to a technical-scientific advance

1 Universidad Autónoma San Sebastian (UASS), San Lorenzo - PY. *E-mail: hendelamaral@outlook.com

2 Universidade de Vassouras (UV), Vassouras - RJ.

3 Universidade de Vila Velha (UVV), Vila Velha - ES.

${ }^{4}$ Centro Universitário FIPMoc (UNIFIPMoc), Montes Claros - MG.

${ }^{5}$ Universidade Federal do Pará (UFPA), Belém - PA.

${ }^{6}$ Centro Universitário de Volta Redonda (UniFOA), Volta Redonda - RJ.

7 Universidade Presidente Tancredo de Almeida Neves (UNIPTAN), São João del Rei - MG.

8 Universidade de Pernambuco (UPE), Serra Talhada - PE.

9 Universidade José do Rosário Vellano (UNIFENAS-BH), Belo Horizonte - MG.

10 PUC Minas (PUC-MG), Poço de Caldas - MG.
} 
that has made possible an optimization of the technique, but also as a result of the fact of having a lower incidence of complications. Although SAVR is still considered the gold standard treatment, TAVR is less invasive and is more suitable for patients at high surgical risk, in addition to presenting similar efficacy and safety to SAVR and better post-surgery prognosis. However, it is essential to analyze other variables such as risk, benefit and comorbidities of the patient before opting for the best treatment technique.

Keywords: Aortic valve stenosis, Surgical aortic valve replacement, Transcatheter aortic valve replacement.

Resumen: Este artículo buscó realizar una revisión de la literatura comparativa entre las técnicas de Reemplazo de la Válvula Aórtica Transcatéter (TAVR) y Reemplazo de la Válvula Aórtica Quirúrgica (SAVR), ambos siendo usados en el tratamiento de la estenosis aórtica, relacionando también las dos técnicas desde el punto de vista del pronóstico y del los indicadores de morbimortalidad. La estenosis aórtica trata del estrechamiento de esta válvula, consistiendo en una condición común de diversas etiologías y puede a menudo evolucionar con hipertrofia ventricular izquierda y tu función disminuida. Actualmente, para el tratamiento de esta patología hubo un aumento en el uso de TAVR, debido no solo a un avance técnicocientífico que ha hecho posible una optimización de la técnica, sino también como consecuencia del hecho de tener una menor incidencia de algunas complicaciones. Aunque se puede considerar el SAVR el estándar de oro, el TAVR es menos invasivo y es más adecuado para pacientes con alto riesgo quirúrgico, además de presentar una eficacia y seguridad similar a SAVR y un mejor pronóstico posquirúrgico. Sin embargo, es fundamental analizar otras variables como el riesgo, el beneficio y las comorbidades del paciente antes de optar por la mejor técnica de tratamiento.

Palabras clave: Estenosis de la válvula aórtica, Reemplazo de la válvula aórtica quirúrgica, Reemplazo de la válvula aórtica transcatéter.

\section{INTRODUÇÃO}

A estenose da válvula aórtica é a causa mais comum de obstrução do fluxo ventricular esquerdo em crianças e adultos e é definida como espessamento e calcificação da válvula aórtica (GAASCH WH, 2020). Se trata de uma condição comum em idosos e a prevalência aumenta com a idade, de $0,2 \%$ para as idades de 50-59 anos a até 10\% para as idades de 80-89 anos (EVEBORN GW, et al., 2013; NKOMO VT, et al., 2006). As três causas principais da estenose aórtica (EA) incluem a válvula congênita anormal (como unicúspide ou bicúspide), frequentemente associada à calcificação, doença cálcica de uma válvula e doença valvar reumática (GAASCH WH, 2020).

Quando a EA se torna hemodinamicamente significativa resulta em resistência à ejeção do ventrículo esquerdo, o que leva a hipertrofia concêntrica como mecanismo compensatório para manter o estresse normal da parede. No entanto, à medida que a gravidade da estenose e a hipertrofia progridem, o ventrículo esquerdo se torna menos complacente e a pressão diastólica final do ventrículo esquerdo aumenta, o que leva a redução da função ventricular (GAASCH WH, 2020). Tendo em vista a fisiopatologia dessa comorbidade, os sintomas mais comuns são: dispneia ao esforço, pré-síncope ou síncope e angina (OTTO CM, 2020).

O tratamento padrão para EA severa é a cirurgia de substituição da válvula aórtica (SAVR), cujas recomendações são baseadas em comparações da história natural dos pacientes com EA aos resultados pós-cirúrgicos (GAASCH WH, 2020). Entretanto, cerca de 30\% dos pacientes não são submetidos ao tratamento em decorrência do elevado risco cirúrgico, principalmente aqueles com idade avançada, disfunção do ventrículo esquerdo e comorbidades associadas (LINDMAN BR, et al., 2014; IUNG B, et al., 2005; BACH DS, 2009).

Nessa perspectiva, uma nova técnica, o implante transcateter da válvula aórtica (TAVR), surgiu como uma alternativa de tratamento menos invasivo a fim de atender os pacientes com estenose aórtica severa de alto risco cirúrgico que são impossibilitados de passar pela substituição valvar cirúrgica (LEON MB, et al., 2010).

$O$ presente artigo tem como objetivo realizar uma revisão de literatura sobre as indicações $e$ contraindicações das técnicas TAVR e SAVR para o tratamento da EA e analisar quais são as diferenças entre os dois métodos em relação ao prognóstico e morbimortalidade. 


\section{REVISÃO BIBLIOGRÁFICA}

O número de procedimentos de TAVR aumentou dramaticamente à medida que grandes estudos de controle randomizados demonstraram sua eficácia e segurança semelhantes em comparação com a SAVR (TOMO MD, et al., 2018).

Existem atualmente 2 sistemas de valva aplicada por cateter em amplo uso clínico para tratar EA: as válvulas que utilizam pericárdio bovino montada em um stent expansível por balão que é colocado inteiramente dentro da valva danificada nativa, e o CoreValve ReValving System que utiliza uma valva pericárdica porcina montada em um stent autoexpansível que se estende até a aorta ascendente para estabilização (WEBB J, et al., 2012).

É importante ressaltar que o sucesso da TAVR depende de uma seleção adequada dos candidatos e da adesão aos critérios de inclusão do protocolo cirúrgico. Dessa forma, é fundamental realizar uma análise do risco cirúrgico e da expectativa de vida do paciente, determinar a viabilidade do procedimento diante da avaliação da anatomia coronariana por cineangiocoronariografia, realizar uma avaliação do acesso vascular e fazer uma adequada avaliação pré-operatória do paciente (ALBAN A, et al., 2020).

Nem todos os estudos atuais concordam com a eficiência da TAVR. Ainda há divergências no meio científico, e elas podem estar associadas aos critérios utilizados em cada pesquisa. Diferentes grupos de idade em cada estudo, comorbidades dos participantes, próteses utilizadas, critérios de inclusão e exclusão e tempo de acompanhamento dos participantes podem interferir nos resultados das pesquisas. Além disso, deve-se levar em consideração que constantemente a técnica e os aparelhos utilizados para o tratamento via transcateter evoluem, e muitos estudos que são contrários à TAVR analisam o procedimento por modelos não mais utilizados atualmente (WANG D, et al., 2019; IMRAN HM, et al., 2018).

Ao contrário do que Gaasch WH (2020) e Siordia JA, et al. (2018) defendem, Tam DY, et al. (2020) e Gaede L, et al. (2017) relatam que em populações com estenose aórtica severa e baixo risco cirúrgico, a TAVR é o procedimento com melhor custo-benefício que a SAVR.

Embora a SAVR ainda seja o tratamento padrão para EA, muitos estudos mostram que 30 a $60 \%$ dos pacientes com essa condição manifestada em uma forma grave são negados ou não são encaminhados para a cirurgia, levando ao uso off-label de TAVR (WANG D, et al., 2019).

Por ainda não ter uma unanimidade no meio científico, é importante que os pacientes sejam sempre informados sobre suas opções de tratamento e os riscos de cada uma. Além disso, é recomendado que seja feita uma abordagem individualizada para cada pessoa, através da incorporação de suas preferências e decisões. Para isso, é necessário que todos os pacientes tenham acesso às duas terapias. No entanto, isso não é possível no Brasil, haja vista que a técnica mais nova, realizada via transcateter, ainda não foi incorporada ao sistema de saúde pública do país; o que vai contra aos princípios sancionados de acesso universal, integral, igualitário e gratuito à saúde (BAVARIA JE, et al., 2019; LOPES MACQ, et al., 2020).

Sabe-se que os avanços na imagem não invasiva e computadorizada, como a tomografia e a ressonância, têm apoiado o crescimento e a maturação do procedimento. Os resultados clínicos melhoraram com a inserção desses equipamentos, devido ao aperfeiçoamento da seleção, da posição do dispositivo e do planejamento individualizado do tratamento para cada paciente (LEETMAA T, et al., 2015).

\section{Indicações}

Para identificar os pacientes que necessitam passar pela substituição de valva, deve-se primeiro identificar seus sintomas. Normalmente dispneia ao se exercitar, síncopes e angina são os sintomas relacionados com a EA. Após isso, deve-se verificar, através de um ecocardiograma, a gravidade da estenose e a fração de ejeção do ventrículo esquerdo. Pacientes que precisam substituir a valva aórtica são os que: têm sintomas relacionados; possuem uma fração de ejeção do ventrículo esquerdo < 50\%; ou não possuem sintomas, mas apresentam uma estenose aórtica severa. De acordo com o autor, estenose aórtica severa pode ser definida como a valva aórtica que tem uma área $\leq 0,75 \mathrm{~cm}^{2}$, além de uma velocidade de jato máxima $\geq 4,5 \mathrm{~m} / \mathrm{s}$ ou um gradiente de pressão transvalvar $\geq 50 \mathrm{mmHg}$ (GAASCH WH, 2020). 
Os profissionais que atuam no atendimento básico de saúde possuem papel fundamental no reconhecimento desses sintomas, pois são eles que devem reconhecer a doença e facilitar o acesso dos pacientes aos procedimentos de substituição de valva encaminhando esses indivíduos para o especialista (BAVARIA JE, et al., 2019).

As duas técnicas possuem vantagens e desvantagens, dependendo da situação. Americanos e europeus recomendam o uso de TAVR em indivíduos com estenose aórtica grave inoperável ou de risco alto para realização de cirurgia (KHAN SU, et al., 2017).

Independentemente de a intervenção ser via cirurgia ou transcateter, se o paciente sentir angina, tiver sinais de isquemia, tiver piora na função ventricular esquerda, tiver histórico de doença coronariana ou tiver fatores de riscos coronarianos (como por exemplo, ser homem $>40$ anos ou mulher após menopausa), ele deve realizar uma angiografia coronariana, pois ele pode durante o procedimento necessitar de uma revascularização coronariana (GAASCH WH, 2020).

\section{Contraindicações}

Foi demonstrada uma associação volume-resultado para procedimentos cardiovasculares como o SAVR. Os volumes do cirurgião têm uma influência significativa nos resultados operatórios em pacientes de alto risco submetidos a SAVR; conforme o volume do cirurgião aumenta, os resultados melhoram de forma correspondente (TOMO MD, et al., 2018). De acordo com Webb J, et al. (2012), os pacientes com risco previsto de morte maior que $50 \%$ ou morbidade irreversível grave eram impossibilitados de realizarem a cirurgia, pois entende-se que a qualidade de vida do paciente não irá mudar e o índice de mortalidade pós cirurgia aumenta significativamente.

Pacientes com disfunção sistólica grave, particularmente na presença de doença coronariana não revascularizada, regurgitação mitral grave ou reserva contrátil pobre, apresentam maior risco de instabilidade hemodinâmica periprocedimento, dessa forma deve-se fazer uma avaliação minuciosa (MASSON B, et al., 2012).

\section{Prognóstico e Mortalidade}

Além de a EA ser uma condição prevalente na população idosa, a sua mortalidade anual chega a $25 \%$. Nesse contexto, o acesso ao tratamento dessa condição deve ser prioridade para reverter esses dados (GLASER N, et al., 2019).

Porém, como Glaser N, et al. (2019) demonstrou, o próprio procedimento de substituição da valva aórtica pode diminuir a expectativa de vida dos pacientes em comparação com a população total, sendo os mais prejudicados pacientes jovens. A própria EA e os casos de cirurgias abertas podem afetar as taxas de sobrevivência dos pacientes.

A implantação de valvas biológicas ou mecânicas pode gerar riscos à curto e à longo prazo, como: hemorragia, derrame, endocardite infecciosa, degeneração da valva, formação de trombos, tromboembolismo, regurgitação aórtica, fibrilação atrial, insuficiência renal aguda, problemas com anticoagulação, necessidade de operações futuras e morte. Sendo que hemorragias, insuficiência renal aguda e fibrilação atrial demonstram ser mais comuns em pacientes que passaram pela SAVR (GLASER N, et al., 2019; GAASCH WH, 2020; SIORDIA JA, et al., 2018).

Tam DY, et al. (2020) comparou três técnicas diferentes para substituição da valva: a tradicional, feita em cirurgia aberta; e as mais novas, as duas vias transcateter, porém uma com um balão expansível e outra autoexpansível. Em relação à mortalidade nos primeiros 30 dias, ao tempo de cirurgia, e à durabilidade da valva, as mais novas tiveram resultados positivos ao comparar com o método tradicional. Em um e dois anos de acompanhamento, não houve diferenças em relação à mortalidade e a derrames (TAM DY, et al., 2020). Além disso, tanto a técnica tradicional quanto as modernas proporcionam uma melhora funcional do coração em 12 meses, e mostraram baixa incidência de trombose, endocardite e necessidade de reoperações (POMPA JJ, et al., 2019; GAEDE L, et al., 2017). De acordo com Imran HM, et al. (2018), as duas técnicas fornecem um aumento na qualidade de vida dos pacientes. 
A frequência de casos em que houve a incompatibilidade de valvas via transcateter é menor que via cirurgia. Porém, os casos de regurgitação da valva aorta, de implementação de marca-passo, de escape valvar e de complicações vasculares são maiores após a TAVR com balões autoexpansíveis (POMPA JJ, et al., 2019; JAWITZ OK, et al., 2020; SIORDIA JA, et al., 2018; TAM DY, et al., 2020).

A necessidade de implantar um marca-passo permanente em um paciente que passou por esse procedimento gera diversas consequências, como o aumento de tempo em UTIs e nos hospitais, e no acréscimo de mais de 10,000 dólares em comparação com os pacientes que não precisaram do aparelho. $O$ escape valvar está associado com um aumento de mortalidade com um ano após as cirurgias (TAM DY, et al., 2020).

Diferente do que outros autores defendem, Little SH, et al. (2016) mostrou que pacientes submetidos à TAVR com prótese autoexpansível possuem menos incompatibilidade com o implante. Também, possuem melhor hemodinâmica da valva e maior área valvar aórtica no período de um ano de análise, quando comparado a SAVR.

Quando as três opções terapêuticas estão disponíveis (balão-expansível, autoexpansível e SAVR) somente após considerar o risco, benefício e as comorbidades de cada paciente - a técnica TAVR com balão expansível demonstra ser a melhor opção na maioria dos casos (TAM DY, et al., 2020; GAASCH WH, 2020).

Um estudo na Alemanha demonstrou em 2016 que as taxas de mortalidade entre os pacientes de médio e alto risco foram significativamente mais baixas entre os que realizaram a TAVR e os que passaram pela SAVR, apenas no grupo de baixo risco que as taxas de mortalidade permaneceram iguais. Além disso, complicações após as substituições de valva também foram menores entre aqueles que passaram pelo procedimento menos invasivo (GAEDE L, et al., 2017).

Candidatos à TAVR considerados risco baixo a intermediário possuem vantagens em relação a sobrevida em médio prazo em relação aos pacientes submetidos a SAVR. No entanto, quando comparados SAVR em pequeno e longo prazo, não há diferença significativa no prolongamento da vida do indivíduo (KHAN SU, et al., 2017).

Uma metanálise feita por Straiton $\mathrm{N}$, et al. (2018) relacionou a TAVR com o aumento significativo na capacidade funcional do coração e na qualidade de vida dos pacientes. De acordo com a pesquisa, esse procedimento pode ter benefícios tanto relacionados à vida do paciente quanto ao coração. Além de diminuir a mortalidade, esse procedimento menos invasivo pode reduzir as limitações físicas que os sintomas da EA causam aos pacientes idosos.

Já outro feito por Makkar RR, et al. (2020) evidenciou que, em grupos com médio risco cirúrgico, pela via transfemoral, a incidência de morte e de derrame cerebral é semelhante entre os pacientes que realizaram a TAVR e a SAVR. Ao comparar com a via transtorácica, a incidência de morte e de derrame cerebral é maior entre os pacientes que passaram pela TAVR. Ele concluiu que, entre as vias de acesso da TAVR, a transtorácica é a que apresenta piores resultados. Além disso, os casos de re-hospitalização e de regurgitação da valva prevaleceram entre aqueles que passaram pelo procedimento menos invasivo.

Em casos de falhas no tratamento pela TAVR, a recuperação pode ser feita por uma substituição "valvein-valve" com uma segunda TAVR ou um reparo cirúrgico por SAVR. A reparação via "valve-in-valve" demonstrou ter menor risco que a SAVR para tratar a deterioração de uma TAVR prévia (JAWITZ OK, et al., 2020).

As causas para que seja preciso realizar futuras cirurgias após o implante são: escape valvar, deterioração estrutural da valva protética, falha no reparo, problemas com tamanho ou posição e endocardite infecciosa. A mortalidade nesses casos foi maior do que o esperado, tanto para os pacientes com baixo risco quanto para os de alto risco cirúrgico (JAWITZ OK, et al., 2020).

Ao comparar pacientes que tiveram que passar por uma reoperação após o procedimento de TAVR e pacientes que foram submetidos desde o início da terapia à SAVR, descobriu-se que os casos de morte póscirúrgico foram mais comuns entre aqueles que tiveram que passar por um segundo procedimento. Não só 
isso, mas a incidência de derrame, insuficiência renal, fibrilação atrial também foi maior neste grupo (JAWITZ OK, et al., 2020).

Em uma pesquisa que ressalta os resultados hospitalares, Ando T, et al. (2018) mostrou que pacientes que realizam TAVR tem menores índices de lesão renal aguda e sangramento que necessite de transfusão, mas possuem mais complicações vasculares. Essa técnica também se mostrou mais vantajosa em hospitais que não são de ensino, gerando melhores desfechos que a SAVR, nessa situação.

SAVR é um procedimento invasivo e está mais associado a encefalopatia após sua execução, quando comparado a TAVR (CONTE JV, et al., 2017). A realização de TAVR resulta em menor risco de AVC em até dois anos, menor tempo de internação hospitalar e de recuperação, por ser menos invasivo (KHAN SU, et al., 2017). Em contrapartida, é um procedimento mais oneroso e com maior regurgitação aórtica paravalvar (ANDO MD, et al., 2018; LITTLE SH, et al., 2016).

Em relação à função ventricular, o volume diastólico final do ventrículo esquerdo mostrou-se reduzido na avaliação precoce dos pacientes após SAVR. Também, ocorreu uma maior disfunção sistólica do ventrículo direito por essa técnica, mas que normalizou em um ano (LITTLE SH, et al., 2016).

As comorbidades que os pacientes apresentam também podem influenciar no prognóstico. Little SH, et al. (2016) mostrou que pacientes que possuem regurgitação paravalvar leve no pré-operatório tanto de SAVR quanto de TAVR possuem menor taxa de mortalidade no período de um ano.

\section{CONSIDERAÇÕES FINAIS}

A partir dos dados supracitados, entende-se que, embora a técnica SAVR ainda se constitua como o procedimento dito padrão para o tratamento da estenose aórtica severa, a técnica TAVR tem se mostrado promissora quanto à eficácia, segurança e custo-benefício em consonância com o método cirúrgico convencional, devido ao seu aspecto menos invasivo e por apresentar melhor prognóstico pós operatório, a exemplo de determinadas características como menor mortalidade apresentada nos primeiros 30 dias, menor tempo de cirurgia, maior durabilidade da valva e menor incompatibilidade com o implante atribuídas ao procedimento. Entretanto, variáveis como adesão aos critérios de inclusão do protocolo cirúrgico, comorbidades e o tempo de acompanhamento dos pacientes após os procedimentos devem ser avaliadas, com o objetivo de elucidar o panorama individual do paciente quanto aos seus riscos e quanto à eficiência de ambas as abordagens cirúrgicas, com enfoque na redução da morbimortalidade destes pacientes.

\section{REFERÊNCIAS}

1. ALBAN A, et al. Implante valvular aórtico transcatéter (TAVI) e impacto en la sobrevida del paciente. Revista Digital de Postgrado, 2020; 9(1).

2. ANDO MD, ADEGBALA et al. In-hospital outcomes of transcatheter versus surgical aortic valve replacement in nonteaching hospitals. Wiley, 2018; 1-6

3. ANDO T, et al. In-hospital outcomes of transcatheter versus surgical aortic valve replacement in non-teaching hospitals. Catheter Cardiovasc Interv. 2019; 93:954-962.

4. BACH DS, et al. Evaluation of patients with severe symptomatic aortic stenosis who do not undergo aortic valve replacement: the potential role of subjectively overestimated operative risk. Circulation: Cardiovascular Quality and Outcomes, 2009; 2(6); 533-539.

5. BAVARIA JE, et al. 2018 AATS/ACC/SCAI/STS Expert Consensus Systems of Care Document: Operator and Institutional Recommendations and Requirements for Transcatheter Aortic Valve Replacement. Journal of the American College of Cardiology, 2019. 73(3): 340-374

6. CONTE JV, et al. Complications After Self-expanding Transcatheter or Surgical Aortic Valve Replacement. Seminars in Thoracic and Cardiovascular Surgery,2017; 29: 321-330.

7. EVEBORN GW, et al. The evolving epidemiology of valvular aortic stenosis. the Tromsø Study. Heart, 2013; 99: 396400 .

8. GAASCH WH. Indications for valve replacement in aortic stenosis in adults. UpToDate, 2020; 1-26.

9. GAASCH WH. Natural history, epidemiology, and prognosis of aortic stenosis Gaasch. UpToDate, 2020; 1-13. 
10. GAEDE L, et al. Outcome after transvascular transcatheter aortic valve implantation in 2016. European Heart Journal, 2017. 39(8):667-675.

11. IMRAN HM, et al. Comparison of phase 2 cardiac rehabilitation outcomes between patients after transcatheter versus surgical aortic valve replacement. European Journal of Preventive Cardiology, 2018. 25(15):1577-1584.

12. IUNG B, et al. Decision-making in elderly patients with severe aortic stenosis: why are so many denied surgery? European Heart Journal, 2005; 26; 2714-2720.

13. JAWITZ OK, et al. Reoperation After Transcatheter Aortic Valve Replacement: An Analysis of the Society of Thoracic Surgeons Database. JACC: Cardiovascular interventions, 2020. 13(13), 1515-1525

14. KHAN SU, et al. Transcatheter vs surgical aortic-valve replacement in low to intermediate-surgical-risk candidates: $A$ meta-analysis and systematic review. Clinical Cardiology, 2017;40:974-981.

15. LEETMAA T, et al. Early aortic transcatheter heart valve thrombosis: diagnostic value of contrast-enhanced multidetector computed tomography. Circ Cardiovasc Interv. 2015; 8(4):1-9.

16. LEON MB, et al. Transcatheter Aortic-Valve Implantation for Aortic Stenosis in Patients Who Cannot Undergo Surgery, 2010; 363(17); 1597-1607.

17. LINDMAN BR, et al. Futility, Benefit and Transcatheter Aortic Valve Replacement. JACC Cardiovascular Interventions, $2014 ; 7$ (7); 707-716.

18. LITTLE SH, et al. Self-Expanding Transcatheter Aortic Valve Replacement Versus Surgical Valve Replacement in Patients at High Risk for Surgery. Circulation: Cardiovascular Interventions, 2016;9:1-17.

19. LOPES MACQ, et al. Tratamento da Estenose Aórtica do Idoso no Brasil: Até Quando Podemos Esperar?. Arquivos Brasileiros de Cardiologia, 2020. 114(2):313-318

20. MAKKAR RR, et al. Five-Year Outcomes of Transcatheter or Surgical Aortic-Valve Replacement. The New England Journal of Medicine, 2020. 382(9): 799-809.

21. NKOMO VT, et al. Burden of valvular heart diseases: a population-based study. Lancet, 2006; 368: $1005-1011$.

22. OTTO CM. Clinical manifestations and diagnosis of aortic stenosis in adults. UpToDate, 2020; 1-23.

23. POMPA JJ, et al. Transcatheter Aortic-Valve Replacement with a Self-Expanding Valve in Low-Risk Patients. The New England Journal of Medicine, 2019. 380(18):1706-1715

24. SIORDIA JA, et al. Three-Year Survival Comparison Between Transcatheter and Surgical Aortic Valve Replacement for Intermediate- and Low-Risk. Innovations (Philadelphia, Pa.), 2018. 13(3):153-162

25. STRAITON N, et al. Functional capacity and health-related quality of life outcomes post transcatheter aortic valve replacement: a systematic review and meta-analysis. Age and Aging, 2018. 47(3):478-482

26. TAM DY, et al. The cost-effectiveness of transcatheter aortic valve replacement in low surgical risk patients with severe aortic stenosis. European Heart Journal-Quality of Care and Clinical Outcomes, 2020. 1-30

27. WANG D, et al. Transcatheter aortic valve implantation versus surgical aortic valve replacement for treatment of severe aortic stenosis: comparison of results from randomized controlled trials and real-world data. Brazilian Journal of Cardiovascular Surgery, 2019. 35(3):346-67 